\title{
Therawhat? Students' Perspectives on the NMT's Role in Theranostic Procedures
}

$\mathbf{T}$

heranostics is a constantly evolving field that is revolutionizing the field of personalized medicine. The term theranostics is derived from the 2 words therapy and diagnostics and is built on the concept that a radionuclide, paired with a chemical compound, can provide both diagnostic and therapeutic components. As nuclear medicine technology students participating in clinical rotations as part of a nuclear medicine technology program, we clearly see that nuclear medicine technologists (NMTs) can have a role in theranostic procedures, whether general or research-based, as they pertain to the field of nuclear medicine technology. A knowledge of and experience with theranostic procedures can prove to be invaluable when it comes to competing in the job market. Moreover, a general knowledge of and background in theranostic procedures can also allow for improved communication with physicians and other hospital personnel to provide patients with the best of care.

A common procedure in which we have seen many NMTs participate is ${ }^{131}$ I-sodium iodide thyroid therapy. It can be considered an example of theranostics, with its 364-keV $\gamma$-emission allowing for diagnostic imaging and its $\beta$-emission providing a therapeutic component (1). Although procedure parameters vary from clinic to clinic, other general procedures that can also be considered theranostic procedures, such as ${ }^{153} \mathrm{Sm}$-lexidronam bone palli-

Received Dec. 19, 2012; revision accepted Jan. 25, 2013.

For correspondence or reprints contact: Miriam E. Van Dyke, University of Alabama at Birmingham, 200 Knoxville Lane, Oxford, AL 36203.

E-mail: mirm@uab.edu

Published online Feb. 12, 2013.

COPYRIGHT (C) 2013 by the Society of Nuclear

Medicine and Molecular Imaging, Inc.

DOI: 10.2967/jnmt.112.118901 ation therapy and ${ }^{90}$ Y-labeled microsphere therapy (SIR-Spheres; Sirtex), have almost always had an NMT component. Whether it is in the follow-up evaluation using a nuclear medicine scan with ${ }^{153} \mathrm{Sm}$ therapy or in the initial biodistribution scan with ${ }^{99 m} \mathrm{Tc}-$ macroaggregated albumin for SIR-Spheres therapy, NMTs are playing a role in theranostic-type procedures (1).

Another molecular breakthrough, which has brought further attention to the field of theranostics, is radioimmunotherapy. Radioimmunotherapy can be described as a theranostic procedure in which antibodies are combined with a therapeutic agent that is a radionuclide with a therapeutic characteristic (e.g., $\beta$-particles). The radionuclide component of the therapy provides not only a therapeutic component but also the ability to image the biodistribution or effectiveness of the therapy. The radionuclide component can be as common as radioiodine, or ${ }^{131} \mathrm{I}$, or as unique as a radiometal (2).

Furthermore, theranostic procedures can entail research procedures that are not considered everyday scans. An example of an investigational procedure that could be considered to uphold the theranostic concept is ${ }^{212} \mathrm{~Pb}-1,4,7,10$-tetra-(2-carbamoyl methyl)-cyclododecane (TCMC) trastuzumab radioimmunotherapy (Areva Med, LLC), which is currently undergoing a clinical phase I trial at the University of Alabama at Birmingham (3). As students who experienced a clinical rotation through the University of Alabama at Birmingham Hospital, we were able to witness NMTs assisting the investigators who are performing the trials. The $\alpha$-particle radioimmunotherapy, which is said to target peritoneal cavity cancers that express HER2, or human epidermal growth factor receptor 2 , required initial ${ }^{99 \mathrm{~m}} \mathrm{Tc}$-macroaggregated albumin scans for peritoneal biodistribution and port patency and ${ }^{212} \mathrm{~Pb}$ follow-up scans (4). NMTs aided investigators in the acquisition of these 2 types of scans. This innovative therapy can be imaged by the emission of $238.6-\mathrm{keV} \gamma$-rays, which are produced during decay of ${ }^{212} \mathrm{~Pb}(5)$. Because many current radionuclide-based therapies use $\beta$-particles, the increasing prevalence of $\alpha$-particle therapies is an exciting development. Because of the inherent traits of $\alpha$-particles, they can potentially decrease the risk of damage to surrounding healthy cells, compared with $\beta$-particles $(2,6)$.

Although NMTs working at universities may have more opportunities to participate in specific investigational theranostic procedures, such as ${ }^{212} \mathrm{~Pb}-$ TCMC-trastuzumab radioimmunotherapy, a basic knowledge of common theranostic procedures such as ${ }^{131} \mathrm{I}-$ sodium iodide therapy can ultimately translate to other theranostic procedures that NMTs may encounter in their career. Although we are currently students, we recognize that it is never too early or too late to take initiative and learn more about the innovative fields, such as theranostic agent production, that are constantly improving and expanding the field of nuclear medicine technology. In addition, as personalized medicine continues to grow with exciting research discoveries, NMTs must heed the call to continue to educate themselves and acquire the skills necessary to provide patients with the highest quality of care possible. Unlike ${ }^{137} \mathrm{Cs}$, not everyone has a long half-life to wait for the next innovative field to develop. With the field of theranostics currently at the back door of many nuclear medicine technology departments, there are endless opportunities to contribute to the field.

\section{DISCLOSURE}

No potential conflict of interest relevant to this article was reported. 


\section{ACKNOWLEDGMENTS}

We acknowledge contributions and advice concerning this editorial from Areva Med, LLC, Ruby Meredith, $\mathrm{MD}, \mathrm{PhD}$, Remo George, MS, CNMT, and Michael T. Azure, PhD.

\author{
Jessica N. Hartline \\ Miriam E. Van Dyke \\ Nuclear Medicine Technology Program
}

University of Alabama at Birmingham

Birmingham, Alabama

\section{REFERENCES}

1. Christian PE, Waterstram-Rich KM, eds. Nuclear Medicine and PET/CT: Technology and Techniques. Maryland Heights. MO: Elsevier Mosby; 2012.

2. Sharkey RM, Goldenberg DM. Cancer radioimmunotherapy. Immunotherapy. 2011;3:349-370.

3. Safety study of ${ }^{212} \mathrm{~Pb}-\mathrm{TCMC}$-trastuzumab radio immunotherapy. ClinicalTrials.gov Web site. http://
clinicaltrials.gov/ct2/show/NCT01384253. Accessed January 28, 2013.

4. Milenic DE, Wong KJ, Baidoo KE, et al. Targeting HER2: a report on the in vitro and in vivo preclinical data supporting trastuzumab as a radioimmunoconjugate for clinical trials. MAbs. 2010;2: 550-564.

5. Pegram M, Slamon D. Biological rationale for HER2/ neu (c-erbB2) as a target for monoclonal antibody therapy. Semin Oncol. 2000; 27(5, suppl 9)13-19.

6. Couturier O, Supiot S, Degraef-Mougin M, et al. Cancer radioimmunotherapy with alpha-emitting nuclides. Eur J Nucl Med Mol Imaging. 2005;32: 601-614. 